\title{
Comparison of the expected and observed supernova remnant counts with Fermi/LAT
}

\author{
Ie. $\operatorname{Vovk}^{1, a}$, A. Neronov ${ }^{2, b}$, and D. Malyshev ${ }^{2, c}$ \\ 1 Max-Planck-Institut für Physik, Föhringer Ring 6, 80805 München, Germany \\ 2 Department of Astronomy, University of Geneva, Ch. d'Ecogia 16, 1290 Versoix, Switzerland
}

\begin{abstract}
SNRs are commonly believed to be the accelerators of the galactic cosmic rays - mainly protons and are expected to produce $\gamma$-rays through the inelastic proton-proton collisions. Fermi/LAT was expected to detect many of those, but only a dozen is listed in the recent Fermi/LAT 2nd Source catalogue. To test whether the observed number of SNRs is in agreement with the above assumption, we use a simplified model of an SNR and calculate the predicted amount of the observable remnants taking into account their distribution in the Galaxy and the sensitivity of Fermi/LAT. We find that the observed number of SNRs agrees with the prediction of our model if we assume a low, $\ll 1 \mathrm{~cm}^{-3}$, number density of the SNR's ambient medium. The result, presented here, suggests, that on average the supernova explosions happen in the under-dense regions, such as bubbles, creating by the winds of the progenitor stars. Under this natural supposition our result finds an agreement with the assumption, that the observed population of supernovae remnants is indeed responsible for the production of the galactic cosmic rays.
\end{abstract}

\section{Introduction}

The supernova remnants (SNRs) - expanding gas shells, resulting from the explosion of the supernovae - are widely observed in the Galaxy. The most up to date catalogue of supernova remnants, composed by [1] and based on the radio observations, lists $\sim 270 \mathrm{SNRs}$.

Being the results of the supernovae explosions, SNRs rapidly expand in the interstellar medium (ISM), producing shock waves where they hit the surrounding gas. Their expansion deposits a very significant amount of energy to their surroundings - on average, every SNR has a kinetic energy of $10^{51}$ ergs at the moment of explosion. This energy is then dissipated in the ISM in various ways - via the hitting of the medium, destruction of the surrounding dense clouds or in the form of the high-energy particles, accelerated within the remnant.

Once escaped, these energetic particles can travel over galactic distances, diffusing in the galactic magnetic fields. These particles are commonly attributed to the galactic part of Cosmic Rays (CR). The exact origin of the galactic cosmic rays is not known, although for decades the SNRs were favoured as their sources due to their energy budget and number in the Galaxy [e.g. 2-4], although alternative models of the $\mathrm{CR}$ production exist [5], related to the acceleration by pulsars and/or pulsar wind nebulae [6] or in the larger scale $\mathrm{OB}$ associations [e.g. 7,8].

Under the assumption that the SNR are the sources of the galactic CRs, the amount of the SNR energy, that goes to the high-energy protons, is not arbitrary and should

a e-mail: Ievgen.Vovk@mpp.mpg.de

b e-mail: Andrii.Neronov@unige.ch

c e-mail: Denys.Malyshev@etu.unige.ch correspond to the observed number of $\mathrm{CR}$ and their leaking rate from the Galaxy. This then translates to the certain average luminosity of the SNRs in the $\gamma$-ray band due to the pion decay.

In what follows we present a statistical, rather than source-by-source, test to verify whether the present quantity of the $\gamma$-ray detected SNRs agrees with the assumption that their population is responsible for production of the galactic cosmic rays.

\section{Estimate of the expected number of the observable SNRs}

To estimate the number of potentially observable supernova remnants in the Galaxy we use a simplified model of the distribution of supernova remnants in the Galaxy. In our analysis the Galaxy is treated as a thin disc with the radius of $15 \mathrm{Kpc}$ and the height, which is much smaller than the thickness. We then apply the following procedure to estimate the number of observable SNRs:

1. characterize a single typical SNR;

2. calculate the total number of the SNR in the Galaxy;

3. distribute this number of SNRs according to their expected spatial distribution in the Galaxy;

4. estimate the sensitivity of the $\gamma$-ray instrument in our possession;

5. calculate the fraction of the SNR population that can be observed with the available $\gamma$-ray telescope.

In our calculations all supernova remnants are considered to be the same and their population is characterized by several parameters, namely the number density of 
the surrounding medium $n_{0}$, average time between the supernova explosions $\Delta T_{S N}$ and a supernova remnant lifetime $T_{\text {life }}$. The $\gamma$-ray luminosity of each remnant $L_{S N R}$ is chosen in a way that the cosmic rays, produced in the remnant, compensate for the observed galactic cosmic ray energy loss $[3,9]$.

To fully characterize the SNR population we also need to specify their distribution in the Galaxy. For this we make the assumption that their distribution follows that of the pulsars, as both pulsars and SNRs are born as a result of the same process - the supernova explosion. Here we assume that their distribution in the galactic plane is axisymmetric and use the radial profile, derived in [10] under the assumption of the clumpy galactic electron distribution, which the authors state to be optimal to describe the observed galactic pulsar population.

To estimate the sensitivity of Fermi/LAT, we used the Fermi/LAT Second source catalogue [11], which lists all the sources detected with Fermi/LAT with more than $4 \sigma$ significance during the first two years of its observations. Our analysis is focused on the Galactic plane, where all the SNRs are situated, which we split into the 30 regions in the Galactic longitude. For each of these regions we select all the sources from the catalogue that lie in the range $b=[-5,+5]$ of the Galactic latitude and chose the faintest as a proxy of the Fermi/LAT sensitivity. We further adjusted it by a factor of $\sqrt{t_{S N R \text { cat }} / t_{2 F G L \text { cat }}} \approx$ $\sqrt{3 / 2}$, which represents the difference in the exposure times between the Second LAT source [11] and the 1st LAT SNR [12] catalogues.

A certain realization of the described SNR population is produced in our calculation chain by randomly distributing $N_{S N R}$ remnants according to the radial profile taken from [10] and then only the SNRs with fluxes above the Fermi/LAT sensitivity in the corresponding direction on the sky are counted. We make a 1000 of such simulations and take the average result as en estimate of the number of the SNRs, that should be observable with Fermi/LAT.

\section{Results and discussion}

The analysis, presented here, aims to test whether the supernovae remnants can be the sources of the galactic component of cosmic rays by comparing the number of the observed SNRs in the high-energy $\gamma$-ray domain with the one expected under this assumption. Recent observations with the Fermi/LAT instrument provided evidences that at least some of the SNRs do accelerate protons to relativistic velocities [13]; however, this does not necessarily mean that all of the supernovae remnants accelerate cosmic rays or do this with the sufficient efficiency.

The soft $(\Gamma \approx-2.6)$ spectrum of cosmic rays suggests, that most of their power is found at the lowest energies. In terms of the $\gamma$-ray flux this means that most of the emission will be produced close to the $\pi^{0}$-production threshold, which corresponds to the photon energies of $E_{\gamma} \approx 140 \mathrm{MeV}$. In this way the Fermi/LAT telescope, sensitive to gamma rays above few hundred GeVs would be best-suited for the detection of this emission.

However, the angular resolution of Fermi/LAT is limited $\left(\sim 1^{\circ}\right.$ at $\left.1 \mathrm{GeV}\right)$. In other words, while being able

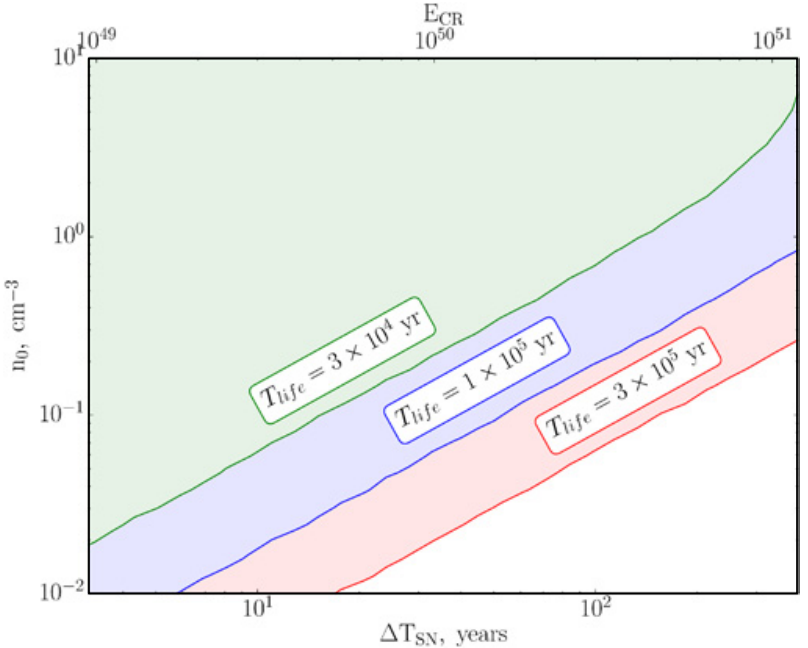

Figure 1. Exclusion plot for the average SNR surrounding density $n_{0}$ and the time between explosions $\Delta T_{S N}$, computed for a set of SNR average lifetimes $T_{\text {life }}$. The exclusion regions corresponds to the $3 \sigma$ significance level.

to detect a source, Fermi/LAT has difficulties in identifying it, if there is a number of possible counterparts. As a result of this limitation, only 19 out of 44 SNRs in the 1st Fermi LAT SNR catalogue [12] are considered as firm detections. This means, that in actuality the number of the detected SNRs lies somewhere between 19 and 44.

The number of the observed SNRs $N_{S N R}^{o b s} \leq 44$ $\left(N_{S N R}^{o b s} \leq 19\right)$ to be compared with our estimates should be further decreased if one takes into account that the emission of some of the observed remnants might be of leptonic, not hadronic, origin. The resulting constraint, stemming from this comparison, is shown in Fig. 1.

For a reasonable estimate of the average SNR lifetime of $10^{5}$ years the predicted number of remnants seats in agreement with observations for $n_{0} \lesssim 1 \mathrm{~cm}^{-3}$ in all range of $\Delta T_{S N}$. If one takes into account the fact that the supernovae explosion rate in our Galaxy is $\sim 30$ years [14], then an immediate conclusion that $n_{0} \ll 1 \mathrm{~cm}^{-3}$ is made. This means, that supernovae predominantly explode in the under-dense regions, where the concentration of particles is much lower than the average in the Galaxy. This conclusion finds agreement with the findings of [15], who did the similar study for the SNRs, detected with the H.E.S.S. Cherenkov telescope.

In this way our analysis indicate that supernovae explode in the under-dense "bubbles", created by their progenitor stars, almost excluding the possibility that $n_{0}$ is $1 \mathrm{~cm}^{-3}$. Given this natural assumption, this reassures the grounds for the hypothesis that SNRs are responsible for galactic cosmic ray population.

\section{References}

[1] D.A. Green, Bulletin of the Astronomical Society of India 37, 45 (2009), 0905. 3699

[2] V.L. Ginzburg, S.I. Syrovatskii, The Origin of Cosmic Rays (1964)

[3] F. Aharonian, A. Bykov, E. Parizot, V. Ptuskin, A. Watson, Space Science Reviews 166, 97 (2012), 1105.0131 
[4] A.M. Hillas, Journal of Physics G Nuclear Physics 31, 95 (2005)

[5] Y. Butt, Nature 460, 701 (2009), 1009. 3664

[6] A. Neronov, D.V. Semikoz, Phys. Rev. D 85, 083008 (2012) 1201.1660

[7] A.M. Bykov, G.D. Fleishman, MNRAS 255, 269 (1992)

[8] J.C. Higdon, R.E. Lingenfelter, and R. Ramaty, ApJL 509, L33 (1998)

[9] V.A. Dogiel, V. Schönfelder, A.W. Strong, A\&A 382, 730 (2002) [arXiv: astro-ph/0111445]

[10] D.R. Lorimer, A.J. Faulkner, A.G. Lyne, R.N. Manchester, M. Kramer, M.A. McLaughlin, G. Hobbs, A. Possenti, I.H. Stairs, F. Camilo, et al., MNRAS 372, 777 (2006), [arXiv: astro-ph/ 0607640]
[11] P.L. Nolan, A.A. Abdo, M. Ackermann, et al., ApJS 199, 31 (2012), 1108.1435

[12] J.W. Hewitt, F. Acero, T.J. Brandt, J. Cohen, F. de Palma, F. Giordano, for the Fermi LAT Collaboration. ArXiv e-prints (2013), 1307.6570

[13] M. Ackermann, M. Ajello, A. Allafort, L. Baldini, J. Ballet, G. Barbiellini, M.G. Baring, D. Bastieri, K. Bechtol, R. Bellazzini, et al., Science 339, 807 (2013), 1302.3307

[14] W. Li, R. Chornock, J. Leaman, A.V. Filippenko, D. Poznanski, X. Wang, M. Ganeshalingam, F. Mannucci, MNRAS 412, 1473 (2011), 1006.4613

[15] P. Cristofari, S. Gabici, S. Casanova, R. Terrier, E. Parizot. MNRAS 434, 2748 (2013), 1302. 2150 